\title{
The periodic behaviour of the North-South asymmetry of sunspot areas revisited
}

\author{
J. L. Ballester ${ }^{1}$, R. Oliver ${ }^{1}$, and M. Carbonell ${ }^{2}$ \\ 1 Departament de Física, Universitat de les Illes Balears, 07122 Palma de Mallorca, Spain \\ e-mail: [dfsjlbQ; ramon.oliver]@uib.es \\ 2 Departament de Matemàtiques i Informàtica, Universitat de les Illes Balears, 07122 Palma de Mallorca, Spain \\ e-mail: marc. carbonell@uib.es
}

Received 16 November 2004 / Accepted 23 December 2004

\begin{abstract}
Up to now, the periodic behaviour of the N-S asymmetry of solar activity has been analyzed by applying the power spectrum analysis to the time series generated from a normalized definition of the asymmetry. Using sunspot areas and the properties of the discrete Fourier transform, we show that the use of the normalized time series leads to misleading results, and that the correct asymmetry time series to be used is generated from the difference between the values of solar activity indicators in the Northern and Southern solar hemispheres. However, in this case the found significant periodicities correspond to periodicities already present in the hemispheric sunspot areas time series, not providing with any interesting information about the asymmetric behaviour of the solar activity. Of course, our conclusions are extensive to all the studies of the periodic behaviour of the N-S asymmetry of solar activity time series computed by means of the normalized definition of the asymmetry, and independent of the considered solar activity feature.
\end{abstract}

Key words. Sun: activity - Sun: sunspots - methods: data analysis - methods: statistical

\section{Introduction}

The north-south (N-S) asymmetry of solar activity has been the subject of many studies carried out using different features of solar activity. Some of the most important indicators considered to undertake these studies have been: the number of flares (Roy 1977; Garcia 1990; Verma 1987, 1992; Li et al. 1998; Temmer et al. 2001), the flare index (Knoška 1985; Ataç \& Özgüç 1996, 1998; Joshi \& Joshi 2004), the sunspot number and sunspot areas (Newton \& Milson 1955; Waldmeier 1957, 1971; Roy 1977; Swinson et al. 1986; Vizoso \& Ballester 1990; Schlamminger 1991; Verma 1992; Yi 1992; Carbonell et al. 1993; Verma 1993; Oliver \& Ballester 1994; Pulkkinen et al. 1999; Li et al. 2000; Li et al. 2002; Vernova et al. 2002; Temmer et al. 2002; Li et al. 2003; Knaack et al. 2004) and the photospheric magnetic flux (Rabin et al. 1991; Knaack et al. 2004). In order to characterize the N-S asymmetry of solar activity, it is usually normalized to the solar activity present in both solar hemispheres and defined as:

$\delta=\frac{N-S}{N+S}$,

where $N$ and $S$ stand for the value of the considered solar activity indicator corresponding to the Northern and Southern hemisphere, respectively. Using this definition, and the power spectrum analysis, different authors have studied the temporal behaviour of the N-S asymmetry of the green coronal line brigthness (Özgüç \& Üçer 1987), soft-X ray flare index
(Joshi \& Joshi 2004), sunspot groups (Verma 1992; Li et al. 2002), sunspot numbers (Watari 1996), sudden disappearances (Vizoso \& Ballester 1989), sunspot areas (Vizoso \& Ballester 1990; Yi 1992; Verma 1992; Carbonell et al. 1993; Oliver \& Ballester 1994; Li et al. 2002; Knaack et al. 2004) and magnetic flux (Knaack et al. 2004) paying attention to the periodicities present in the considered N-S asymmetry time series.

Applying Eq. (1) to sunspot areas and using the power spectrum analysis, many studies of the periodic behaviour of the $\mathrm{N}-\mathrm{S}$ asymmetry of sunspot areas have been performed. These studies agree in pointing out the presence of a significant periodicity having a long period (Vizoso \& Ballester 1990; Verma 1992; Carbonell et al. 1993; Oliver \& Ballester 1994; Oliver \& Ballester 1996; Pulkkinen et al. 1999; Li et al. 2002; Knaack et al. 2004), as well as another significant periodicity around eleven-twelve years (Carbonell et al. 1993; Oliver \& Ballester 1994; Oliver \& Ballester 1996; Li et al. 2002; Knaack et al. 2004). Furthermore, other periodicities claimed to be significant have been also reported (Knaack et al. 2004). However, Yi (1992) suggested that the presence of the periodicity around eleven-twelve years could be produced by the denominator of the normalized asymmetry, which represents the total solar activity.

In this Letter, our aim is to show that the results obtained by the application of the power spectrum analysis to the normalized asymmetry time series obtained from Eq. (1) are misleading, not only regarding the peak around eleven years, but 

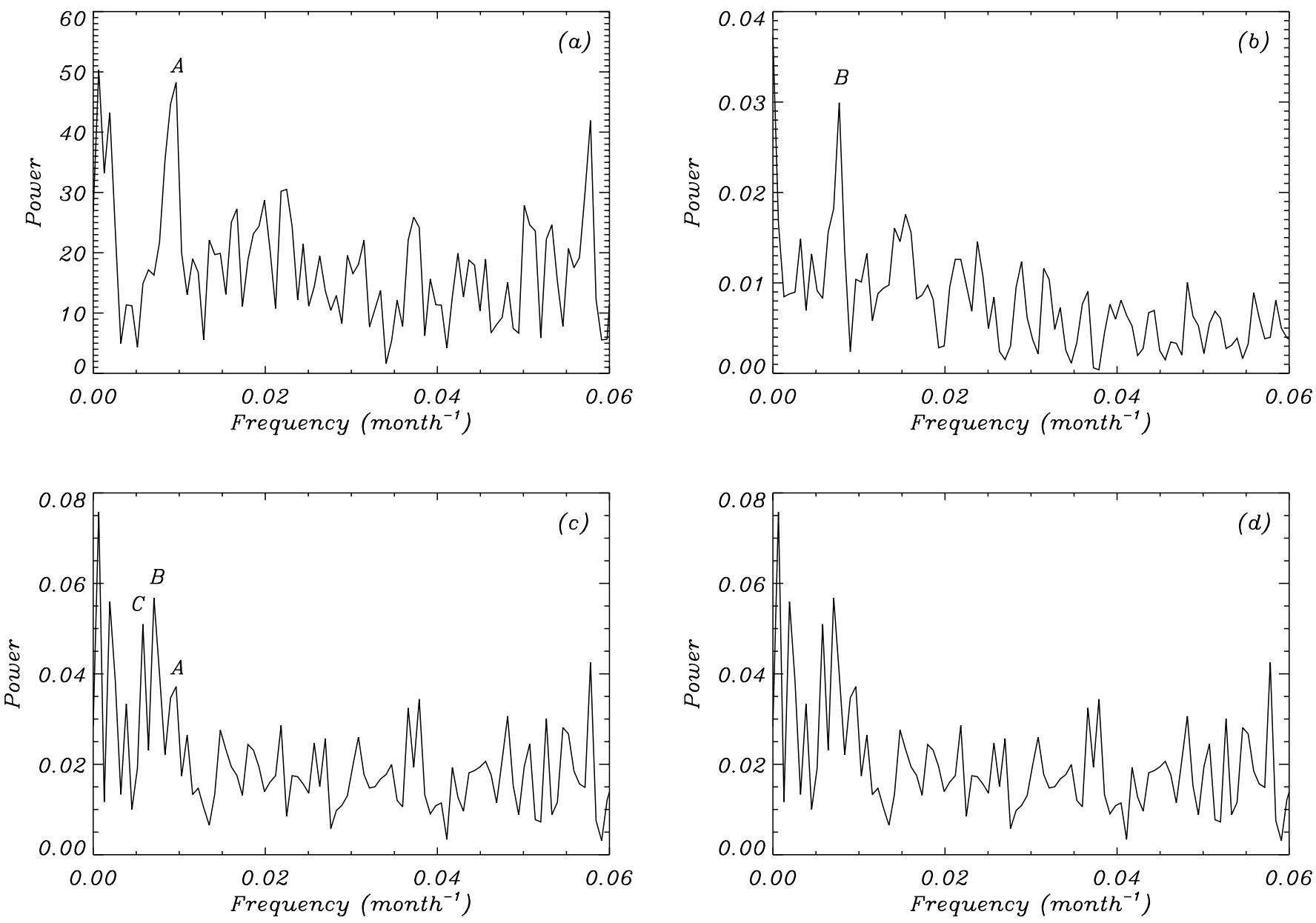

Fig. 1. Discrete Fourier Transform of a) the difference between Northern and Southern hemispheric sunspot areas $(N-S)$; b) the inverse of the total sunspot area $\left.\left(\frac{1}{N+S}\right) ; \mathbf{c}\right)$ the normalized North-South asymmetry of sunspot areas $\left.\left(\frac{N-S}{N+S}\right) ; \mathbf{d}\right)$ circular convolution of a) and b).

also with respect to the general shape of the power spectrum, and that another definition of the asymmetry must be used to study its periodic behaviour.

\section{Data, methods and results}

The data used in our study are the monthly sunspot area data (5/1874-1/2004), compiled by D. Hathaway, and which can be downloaded from http://science.msfc.nasa.gov/ $\mathrm{ssl} / \mathrm{pad} / \mathrm{sol}$ ar/greenwch.htm. These data provide us with two different time series for the Northern and Southern solar hemispheres, composed of 1557 values. From the properties of the Discrete Fourier Transform (DFT) we know that the DFT of a product of two $N$-point sequences is the circular convolution of their respective DFTs. In particular, if $x_{3}=x_{1} x_{2}$ then $X_{3}[k]$, the DFT of $x_{3}$, is

$X_{3}[k]=\frac{1}{N} \sum_{l=0}^{N-1} X_{1}[l] X_{2}[k-l(\bmod N)]$,

with $X_{1}[k]$ and $X_{2}[k]$ the DFTs of $x_{1}$ and $x_{2}$, respectively (Oppenhein \& Schafer 1989). Now, going back to Eq. (1), $x_{3}$ can be identified with $\delta$, while $x_{1}$ and $x_{2}$ correspond to $N-S$ and $\frac{1}{N+S}$, respectively. Then, the DFT of $\delta$ is the circular convolution of the DFTs of $N-S$ and $\frac{1}{N+S}$, and is obtained using Eq. (2).

In Fig. 1 we show the more relevant part of the computed DFTs corresponding to the frequency interval up to 0.06 month $^{-1}$. For instance, in Fig. 1a we can see an important peak (A) at a period of 8.65 years while in Fig. 1b a significant peak (B) appears at a period of 10.91 years, which corresponds to the solar cycle. However, once the convolution has been performed, Fig. 1c shows that these peaks appear within a sequence of three peaks $(\mathrm{C}, \mathrm{B}, \mathrm{A})$ at periods of $14.4,11.79$ and 8.65 years, respectively. Then, the peaks B, A corresponding to different DFTs appear together in Fig. 1c, although the frequency of peak $\mathrm{B}$ has been slightly modified by the convolution. A new peak $\mathrm{C}$ which was not relevant in Figs. 1a or b also appears. Finally, Fig. 1d fully agrees with Fig. 1c as expected. This analysis points out clearly that the suggestion made by Yi (1992) is completely right and that the peak at 11.79 years appearing in the power spectrum of the asymmetry time series generated by means of Eq. (1) comes from the denominator, i.e. from the joint solar activity of both hemispheres. Of course, this analysis can be extended to the rest of peaks appearing in panels (c) or (d) and points out that, apart from peaks coming from solar activity, other peaks coming from the circular convolution also appear. Then, in general, the periodic behaviour 
of the N-S asymmetry of solar activity cannot be studied by computing the power spectrum of the time series generated using the normalized definition of asymmetry given by Eq. (1).

On the contrary, using again the properties of the DFT, we know that given $x_{1}$ and $x_{2}$ the DFT of $x_{1}-x_{2}$ is given by $X_{1}[k]-X_{2}[k], X_{1}[k]$ and $X_{2}[k]$ being the DFTs of $x_{1}$ and $x_{2}$, respectively. Then, we use as definition of the N-S asymmetry of solar activity,

$\Delta=N-S$,

expressing the difference between both hemispheres but without any normalization. Consequently, we can study the periodic behaviour of the time series generated using Eq. (3) by computing the DFT of $\Delta$, which is given by

$\Delta[k]=N[k]-S[k]$,

where $\Delta[k], N[k]$ and $S[k]$ are the DFTs of $\Delta, N$ and $S$, respectively. The important difference of Eq. (4) with respect to Eq. (2) is that the DFT of the N-S asymmetry, $\Delta$, is obtained from a direct sum of DFTs computed at the same frequency and without any convolution.

Taking into account that $\Delta[k]$ has already been computed, we could study its periodic behaviour by analyzing the power spectrum shown in Fig. 1a. However, to perform the study we have chosen the Lomb-Scargle periodogram (Lomb 1976; Scargle 1982; Horne \& Baliunas 1986), in which the power $P_{N}(\omega)$ is defined so that if the signal is pure noise (with zero mean and standard deviation $\sigma=1$ ), then the power $P_{N}(\omega)$ follows an exponential probability distribution. This fact is very useful, because it means that for any frequency $\omega_{0}$ the probability that $P_{N}\left(\omega_{0}\right)$ is of height $z$ or higher is $\mathrm{e}^{-z}$. Then, we can use the False Alarm Probability (FAP) formula to estimate the statistical significance of a peak in the power spectrum. The FAP is given by

$F=1-\left[1-\mathrm{e}^{-z_{\mathrm{m}}}\right]^{N}$,

where $z_{\mathrm{m}}$ is the height of the considered peak and $N$ is the number of independent frequencies. Fourier components calculated at frequencies at intervals of the independent Fourier spacing, $\Delta f_{\text {ifs }}=1 / t$, where $t$ is the time span of the data, are totally independent (Scargle 1982). Then, the periodogram has been computed for exactly the same Fourier frequencies as the DFT, and in this way the number of independent frequencies is fixed and corresponds to 778 . Figure 2 shows the periodogram for the $\mathrm{N}-\mathrm{S}$ asymmetry, $\Delta$, in the frequency interval up to 0.06 month $^{-1}$ and four relevants peaks $(\alpha, \beta, \gamma$ and $\epsilon$ ) appear at periods of $129.75,43.25,8.65$ and 1.44 years, respectively. The apparition of a peak $(\alpha)$ at a period corresponding to the span of the time series suggests the presence of an underlying trend in the time series. Then, we have performed a Cox-Stuart test whose statistic $z$ gives a value of 4.08 and confirms that the null hypothesis of trend presence can be accepted with an error probability smaller than $0.1 \%$. The time series can be detrended by subtracting a second order polynomial from the time series, which removes the power at very long periods and, then, the Cox-Stuart test gives a value of the $z$ statistic of 0.13 , which is statistically insignificant. Then, the peak at long period is a

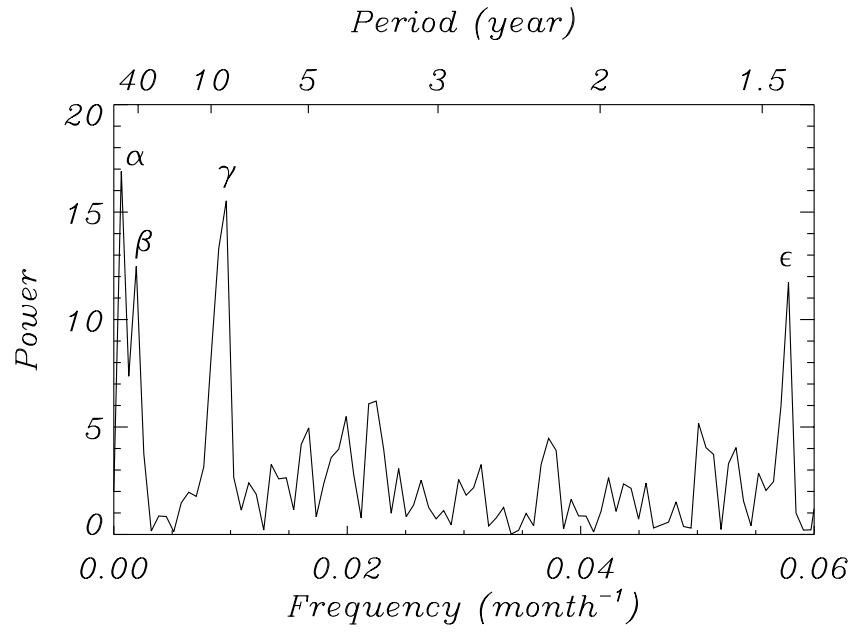

Fig. 2. Lomb-Scargle periodogram of the N-S asymmetry of sunspot areas, $\Delta$, computed as the difference between the Northern and Southern hemispheric sunspot areas. Peak $\alpha$ corresponds to the underlying trend of the time series, while peaks $\beta, \gamma$ and $\epsilon$ have periods of $43.25,8.65$ and 1.44 years, respectively.

spurious periodicity produced by the underlying trend of the time series. Now, we can estimate the FAP for the remaining peaks $\beta, \gamma$ and $\epsilon$ whose value is of $0.0028,0.00023$ and 0.0047 , respectively, i.e. the probability to obtain these peaks by chance is $0.28 \%, 0.023 \%$ and $0.47 \%$. The remaining peaks in the periodogram have very low power, giving (according to the FAP) very high probabilities to be obtained by chance. Then, these three peaks constitute the only significant periodicities appearing in the N-S asymmetry of sunspot areas time series computed following Eq. (3). On the other hand, and as a further confirmation that the periodogram gives the same results as the DFT, comparing Figs. 2 with 1a we can see that both power spectra show the same shape with the three significant peaks, as well as the trend.

Now, the remaining task is to understand the origin of these periodicities in the N-S asymmetry of sunspot areas. This can be easily understood by performing periodograms of the sunspot areas time series corresponding to the Northern and Southern hemispheres. In these periodograms it can be seen that the significant peaks found in the periodogram of the $\mathrm{N}-\mathrm{S}$ asymmetry, $\Delta$, are already present although they are very weak as compared to the peak corresponding to the solar cycle. However, this peak and another one at a period slightly longer than that of the solar cycle, appearing in both periodograms, are missing in the periodogram of the N-S asymmetry $\Delta$ (Fig. 2). The reason for this behaviour is that both periodicities occur in both hemispheres at exactly the same period and in phase, so, when we perform the Fourier analysis using Eq. (4) these periodicities cancel. The cancellation of the periodicity corresponding to the solar cycle allows other periodicities to become significant in the resulting periodogram. For instance, in the case of the southern hemisphere, apart from the solar cycle peak, the second strongest peak corresponds to 8.65 years, which also appears as the most significant in the power spectrum of the N-S asymmetry, $\Delta$. 
Further confirmation can be obtained from the wavelet analysis, which shows that the epoch of activity of the significant periodicities in the N-S asymmetry of sunspot areas time series coincides with the epochs in which those periodicities have been active in one or both solar hemispheres.

\section{Conclusions}

Up to now, the study of the periodic behaviour of the N-S asymmetry has been undertaken by performing a DFT of the N-S asymmetry time series obtained from the normalized definition of asymmetry (Eq. 1). This procedure is equivalent to computing the circular convolution of the DFTs corresponding to the $N-S$ and $\frac{1}{N-S}$ time series, which introduces peaks coming from the periodic behaviour of solar activity represented by the denominator, as well as others coming from the circular convolution (Fig. 1). Taking into account the properties of the DFT, it seems that the right way to study the periodic behaviour of the N-S asymmetry of solar activity is to use the time series generated by $N-S$, which accounts for the difference between solar activity in both hemispheres.

Applying this procedure to sunspot areas, the Fourier analysis leads to the following conclusions: (1) The high peak which appears at long periods in the power spectrum is not a true periodicity. It appears at a period corresponding to the span of the time series (and so can never be longer than the considered time series as in Knaack et al. 2004) and is a typical feature suggesting the presence of an underlying long term trend in the time series. It can be easily detected using simple statistical tests against trends and can be characterized by fitting polynomials of different order to the considered time series. Finally, the time series can be detrended by subtraction of the fitted polynomial or by differencing; (2) Only three significant peaks appear in the power spectrum with periods of $43.25,8.65$ and 1.44 years; (3) These found significant periodicities are present in one or both hemispheric sunspot areas time series.

Previous studies of the periodic behaviour of the normalized N-S asymmetry time series agree about the presence of two significant periodicities, one having a very long period and another around 11 years. However, following our approach, the only coincident periodicity is that having a long period, while the periodicity at 11 years does not appear, which confirms the suggestion made by Yi (1992). The rest of significant periodicities in our study are already present in the Fourier analysis of sunspot areas time series of one or both solar hemispheres.

Then, our study leads to two main conclusions: (1) that the study of the periodic behaviour of the N-S asymmetry of sunspot areas must be performed by Fourier analysing the time series obtained from Eq. (3), which avoids the appearance of spurious peaks coming from the circular convolution of the DFTs corresponding to the factors of Eq. (1); (2) that following this approach, the found significant periodicities, apart from the long term trend, correspond to periodicities already present in the Fourier analysis of hemispheric sunspot areas. Then, there is not much interest in the study of the periodic behaviour of the N-S asymmetry of sunspot areas, and what is really important is to quantify the excess of the activity of one hemisphere with respect to the other, as well as the statistical significance of this excess, which already was done in the past (Vizoso \& Ballester 1990; Carbonell et al. 1993)

Of course, the conclusions presented here about how to study the periodic behaviour of the N-S asymmetry of sunspot areas are extensive to any considered feature of solar activity (magnetic flux, solar flares, etc.)

Acknowledgements. The authors acknowledge the helpul suggestions received from Biel Cardona.

\section{References}

Ataç, T., \& Özgüiç, A. 1996, Sol. Phys., 166, 201

Ataç, T., \& Özgüç, A. 1998, Sol. Phys., 180, 397

Carbonell, M., Oliver, R., \& Ballester, J. L. 1993, A\&A, 274, 497

Garcia, H. 1990, Sol. Phys., 127, 185

Horne, J. H., \& Baliunas, S. L. 1986, ApJ, 302, 757

Joshi, B., \& Joshi, A. 1992, Sol. Phys., 219, 343

Knaack, R., Stenflo, J. O., \& Berdyugina, S. V. 2004, A\&A, 418, L17

Knoška, S. 1985, Contrib. Astron. Obs. Skalnat Pleso, 13, 217

Li, K. J., Schmieder, B., \& Li, Q. Sh. 1998, A\&AS, 131, 99

Li, K., Gu, X., Xiang, F., Liu, X., \& Chen, X. 2000, MNRAS, 317, 897

Li, K. J., Wang, J. X., Xiong, S. Y., et al. 2002, A\&A, 383, 648

Li, K. J., Wang, J. X., Zhan, L. S., et al. 2003, Sol. Phys., 215, 99

Lomb, N. 1976, Ap\&SS, 39, 447

Newton, H. W., \& Milson, A. S. 1955, MNRAS, 115, 398

Oliver, R., \& Ballester, J. L. 1994, Sol. Phys., 152, 481

Oliver, R., \& Ballester, J. L. 1996, Sol. Phys., 169, 215

Oppenhein, A. V., \& Schafer, R. W. 1989, Discrete-time Signal Processing (Prentice Hall), 547

Özgüç, A., \& Üçer, C. 1987, Sol. Phys., 114, 141

Pulkkinen, P. J., Brooke, J., Pelt, J., \& Tuominen, I. 1999, A\&A, 341, L43

Rabin, D. M., DeVore, C. R., Sheeley, N. R., Harvey, K. L., \& Hoeksema, J. T. 1991, in Solar Interior and Atmosphere, ed. A. C. Cox, W. C. Livingston, \& M. S. Matthews (The Universe of Arizona Press), 781

Roy, J. R. 1977, Sol. Phys., 52, 53

Scargle, J. D. 1982, ApJ, 263, 385

Schlamminger, L. 1991, Sol. Phys., 135, 407

Swinson, D. B., Koyama, H., \& Saito, T. 1986, Sol. Phys., 106, 35

Temmer, M., Veronig, A., Hanslmeier, A., Otruba, W., \& Meserotti, M. 2001, A\&A, 375, 1049

Temmer, M., Veronig, A., \& Hanslmeier, A. 2002, A\&A, 390, 707

Verma, V. K. 1987, Sol. Phys., 114, 185

Verma, V. K. 1992, in The Solar Cycle, ed. K. Harvey, ASP Conf. Ser., 31,429

Verma, V. K. 1993, ApJ, 403, 797

Vernova, E. S., Mursula, K., Tyasto, M. I., \& Baranov, D. G. 2002, Sol. Phys., 205, 371

Vizoso, G., \& Ballester, J. L. 1989, Sol. Phys., 112, 317

Vizoso, G., \& Ballester, J. L. 1990, A\&A, 229, 540

Waldmeier, M. 1957, ZAp, 43,149

Waldmeier, M. 1971, Sol. Phys., 29, 232

Watari, S. 1996, Sol. Phys., 163, 259

Yi, W. 1992, JRASC, 86, 2, 89 